\title{
Biological Feedback Controller Design for Handwriting Model
}

\author{
Mariem BADRI ${ }^{1}$ Afef ABDELKRIM ${ }^{3}$ \\ Laboratory of Automation Research (LA. RA), (ENIT) \\ National School of Engineering of Carthage (ENICarthage) \\ Tunis, Tunisia
}

\author{
Ines $\mathrm{CHIHI}^{2}$ \\ Laboratory of Automation Research (LA. RA) \\ National School of Engineering of Tunis \\ Tunis, Tunisia
}

\begin{abstract}
This paper deals with a feedback controller of PD (proportional, derivative) type applied to the process of handwriting. The considered model for this study describes the behavior of the system "hand and pen" to forearm muscles forces, applied for the production of handwriting. The applied approach considers memory recall of error signal between model outputs and experimental data to reach a desired trajectory position, a rapid dynamic and stable model response. The control technique is applied in order to expand the handwriting model response to a larger database of graphic traces. The obtained results illustrated the reliability of closed loop control to command the handwriting system, and to ensure its robustness against unknown inputs such as muscles forces that could vary from an individual to another and increase model complexity.
\end{abstract}

Keywords-Handwriting system; biological system; feedback control; PD controller; muscles forces signals

\section{INTRODUCTION}

Hand loss occurs due to different causes and has been increased in many countries. It has without exception, profound economic psychological and social impacts. A hand serves many roles. It is acting as a multifunctional tool for interacting with the world. Thus, prosthesis hand is vital for everyone that is in need of it. However, most of the proposed prosthesis offer limited and simple functions as opening, closing, grasp objects by pinching type, etc. They are unable to perform movements requiring some precision such as handwriting with conserving the individual characteristics of the writer (roundness or sharpness, inclination, regular or irregular spacing between letters, etc.). These characteristics are maintained even when we write with body segments as incongruous as the mouth and the foot.

In [1-3], the increase in functionality of hand prosthesis is mainly based on the progression of the control strategies. The most used control approaches are based on the amplifier electrical activity of the muscles, named ElectroMyoGraphy signal (EMG) which allows encoding directly the orders generated by the brain or muscles forces. These signals are strongly related to the muscles forces as mentioned in [4,5] which are used in several hand's characterisation approaches. Indeed, [6] used these forces to develop a highly realistic human hand and forearm model. In 2015, [7] proposed a stable force-myographic control for prosthetic hand using incremental learning. Robust hand force estimation is also studied recently in [8].
Other researches such as [9-12] show that the complex movement of hand writing can be modelled on the plane $(x, y)$, from two most active forearm muscles, named "Abductor Pollicis Longus" and "Extensor Carpi Ulnaris". They assure vertical and horizontal displacement, respectively. The control theories are based on two electromyography signals EMG1 and EMG2 of these forearm muscles.

In order to refine the proposed handwriting models in the literature, we propose to ameliorate the mathematical model proposed in [12]. The biological process is compared, in this work, to a dynamic system model described by a set of two nonlinear differential equations. It is controlled by the muscles forces and incorporates viscosity coefficient and a frictional force applied during writing act between the pen tip and the writing plant.

The main contribution of this paper is to model handwriting motion by a feedback controller wildly used in literature [1315]. Quite recently, a considered attention has been paid to field of biomedical control system and a growing interest gave emerged for applying feedback controller in this area. In [16], researchers focused on the simulation of reflexive muscle. Literature in [17] was devoted to the development of automotive safety system, active muscles response were implemented using feedback control of a nonlinear model of human arm.

In this paper, the considered controller is of PD type (i.e. Proportional and derivative). Indeed, the purpose is to reduce modelling error and improve system stability to the studied uncertain inputs, due to their nature, and increase the model dynamic. Actually, in the considered model we allow the integration of the human expertise decision in the studied system during the human handwriting motion, different elements react and intervene in the same time to produce homogeneous writing, readable and without deviation, such as the system of perception, the brain, the muscles, etc. In this context, $[18,19]$ presented a generalized handwriting model and proved that the pen-tip position is detected by the eyes. Information are transmit to the brain for analyse and comparison with the desired position. Then, electrical activities are sent to muscles to execute the desired writing movement.

The proposed handwriting model can be applied in several areas, such as, trajectory generator for hand-robot control, neuro-prosthetic devices; rehabilitation practices and even to in diagnose of neurological disease like Parkinson and Alzheimer. 
The paper is organized as follows; Section II is devoted to the experimental approach proposed for characterizing the handwriting system. Section III reports the studied model and control of handwriting system. Section IV discusses the background of our study by outlining a feedback control technique of PD type applied to control function of handwriting model, and illustrated by simulation results and analysis. Finally section V concludes with a summary.

\section{EXPERIMENTAL APPROACH}

In order to characterize handwriting movements, an experimental approach was proposed in [9], in Hiroshima City University, to record at the same instant cursive Arabic letters or geometric forms and two forearm muscles EMG signals. The measuring data were synchronized by sending a step signal from the parallel interface port on the computer to the data recorder. This experimentation had required the following equipment:

- Digital table of the brand "WACOM, KT-0405-RN".

- Pre-amplifiers "TEAC, AR-C2EMG1".

- Data recorder "TEAC, DR-C2".

- Bipolar surface electrodes (MEDICOTEST, Blue Sensor N-00-S).

- Computer.

Fig. 1 indicates the positioning of electrodes on the writer's arm. Electrodes, indicated by "ch1", is relative to the first muscle and those relative to the second muscle are indicated by "ch2".
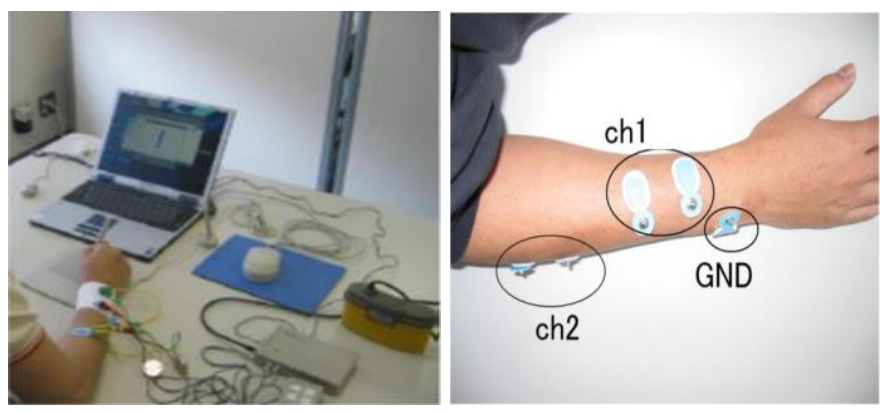

Fig. 1. Experimental assembly and electrodes' positions.
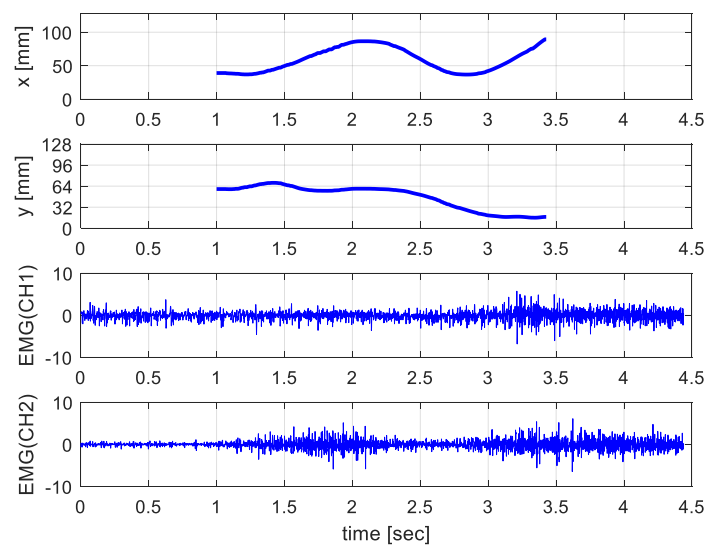

(a)
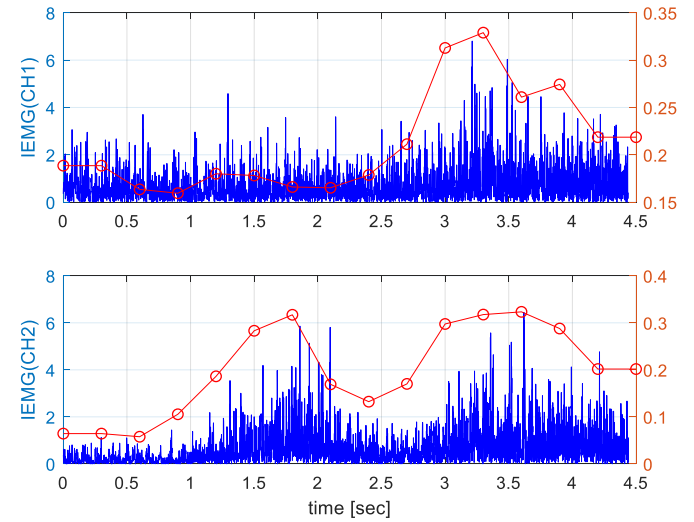

(b)

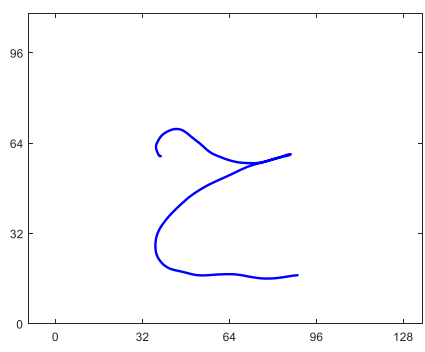

(c)

Fig. 2. The letter, $ح$, «HA» (a) Movement on the plane (x,y) and EMG signals (b) IEMG signals (c) Form.

In Fig. 2, are presented the recorded data for the Arabic letter, ح, "HA", from [10]. It is difficult to get the useful information from muscles activities. For this, a variety of signal processing techniques are used to make EMG waveforms easier to interpret. Indeed, the fluctuation of EMG's magnitudes can be filtered, [10], to obtain new curves called Integrated EMG (IEMG), represented by dotted red curves in Fig. 2 (b).

TABLE I. CONSIDERED ARABIC LETTERS AND GEOMETRIC ForMS

\begin{tabular}{|l|l|l|l|l|l|}
\hline $\mathrm{Nb}$ & $\begin{array}{l}\text { Shape } \\
\text { description }\end{array}$ & Shape & $\mathrm{Nb}$ & $\begin{array}{l}\text { Shape } \\
\text { description }\end{array}$ & Shape \\
\hline 1 & $\begin{array}{l}\text { Horizontal } \\
\text { line(1)(left/right/left) }\end{array}$ & $\begin{array}{l}\text { Circle (2) } \\
\text { (to the } \\
\text { left) }\end{array}$ & \\
\hline 2 & $\begin{array}{l}\text { Horizontal line (2) } \\
\text { (right/ left / right) }\end{array}$ & 7 & $\begin{array}{l}\text { Triangle } \\
\text { (1) (to the } \\
\text { right) }\end{array}$ & \\
\hline 3 & $\begin{array}{l}\text { Vertical line } \\
\text { (1)(up/down/up) }\end{array}$ & $\begin{array}{l}\text { Triangle } \\
\text { (2) (to the } \\
\text { left) }\end{array}$ & \\
\hline 4 & $\begin{array}{l}\text { Vertical line (2) } \\
\text { (down/ up / down) }\end{array}$ & 9 & $\begin{array}{l}\text { Arabic } \\
\text { letter(SIN) }\end{array}$ & \\
\hline 5 & $\begin{array}{l}\text { Circle (1) } \\
\text { (to the right) }\end{array}$ & 0 & 10 & $\begin{array}{l}\text { Arabic } \\
\text { letter (HA) }\end{array}$ & \\
\hline & & 11 & $\begin{array}{l}\text { Arabic } \\
\text { letter } \\
\text { (AYN) }\end{array}$ & \\
\hline
\end{tabular}


Scripters were of different gender and ages. The data base is a set of some Arabic letters, and eight basic geometric shapes, presented in table I of [9].

\section{SYSTEM DESCRIPTION}

The handwriting motion process is based on the concept of memory, eyes recognition, analysis and decision made by the brain achieved by an order for muscles excitation. Fig.3 illustrates this process with the eyes as a sensor (Block 3 ) detecting the current pen tip position (coordinates in the plane $(x, y))$ during the writing motion. Then, the biological sensor sends information to the brain (Block 4). The analysis and evaluation of this is achieved by a decision and sending an order in the form of muscle activities (EMG). These signals will load the forearm muscles (Block 1) to control the arm, hand (Block 2), and execute the desired writing movement.

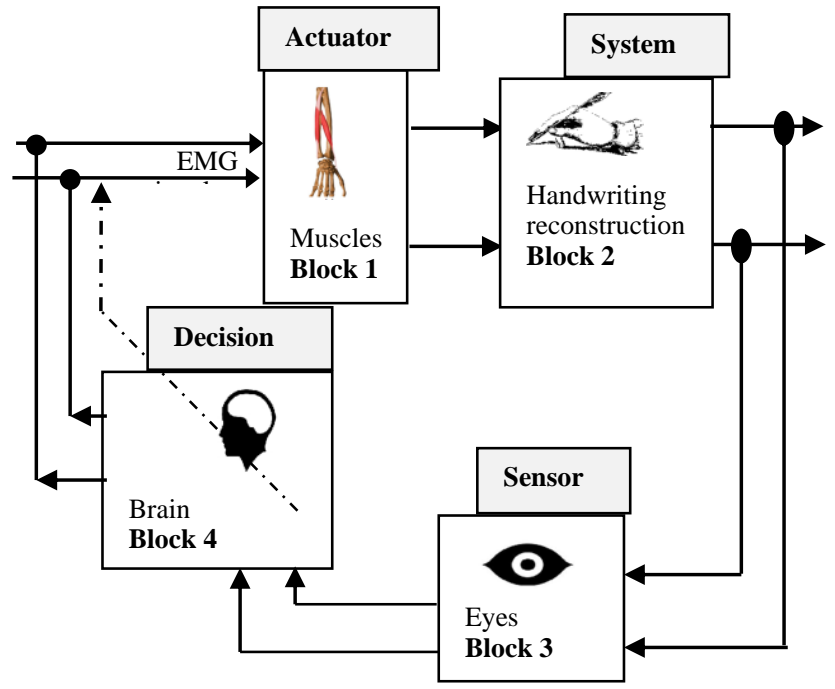

Fig. 3. Biological feedback controller design.

As we mentioned, the proposed study is an amelioration of the handwriting model presented by Yasuhara in [12]. This work takes into account the effect of stiffness relative to the hand viscosity and frictional force between the pen-tip and the writing surface, for a considered mass $\mathrm{M}$, equivalent to the mass of the scripter hand and the pen. This model (Block 2 of Fig.4) is expressed by equations (1) to (3).

$\ddot{d}=F_{d}-k_{d} \dot{d}$

$k_{d}=\lambda_{d}+\mu_{d} \frac{p}{v}$

$v=\sqrt{\left(\dot{x}^{2}+\dot{y}^{2}\right)}$

With

$d \quad$ : the graphic trace coordinates corresponding to $\mathrm{x}$ and y axis,

$F_{d}$ : the equivalent force applied by the muscles on the mass $\mathrm{M}$, $k_{d}$ : the viscosity coefficient expressed in (2) with :

$\lambda_{d} \quad:$ the internal hand viscosity coefficient equivalent to a mass $\mathrm{M}$,

$\mu_{d}:$ the equivalent stiffness coefficient between the pen-tip and writing surface,

$p \quad:$ the equivalent pressure applied by the pen-tip on the writing surface,

$v \quad$ : the writing velocity

The parameters $\lambda_{d}$ and $\mu_{d}$ impose the dynamic of writing, and this make possible the characterizing of each writer, but also increases the model complexity.

After the estimation of these parameters and assuming that the pressure is constant, the equation in (1) could be expressed as in (4).

$$
\left\{\begin{array}{l}
\ddot{x}=F_{x}-\left(4.7+\frac{0.75}{\sqrt{\left(\dot{x}^{2}+\dot{y}^{2}\right)}}\right) \dot{x} \\
\ddot{y}=F_{y}-\left(4.7+\frac{0.75}{\sqrt{\left(\dot{x}^{2}+\dot{y}^{2}\right)}}\right) \dot{y}
\end{array}\right.
$$

Where $x$ and $y$ are the coordinates of the pen-tip movement on a biaxial plane. The viscosity coefficient is expressed as $k_{d}=\left(4.7+\frac{0.75}{\sqrt{\left(\dot{x}^{2}+\dot{y}^{2}\right)}}\right)$, the non-linearity of this model is a function of the writing velocity $v=\sqrt{\left(\dot{x}^{2}+\dot{y}^{2}\right)}$,

$F_{x}$ and $F_{y}$ are the muscles force applied to a mass $M$ corresponding to hand movement on $x$ and $y$ axis, respectively.

From the differential equation system in (4), it's a $4^{\text {th }}$ order system and we choose:

$$
\begin{aligned}
& z \in \mathbf{R}^{4} \text { is the state vector: } \\
& z=\left(\begin{array}{l}
z_{1} \\
z_{2} \\
z_{3} \\
z_{4}
\end{array}\right)=\left(\begin{array}{c}
x \\
\dot{x} \\
y \\
\dot{y}
\end{array}\right)=\left(\begin{array}{c}
\text { movement } \text { on } \text { x axis } \\
\text { velocity } \text { on } \text { x axis } \\
\text { movement on y axis } \\
\text { velocity on y axis }
\end{array}\right)
\end{aligned}
$$

The state representation of the handwriting model is described in (5).

$$
\dot{z}=\left(\begin{array}{c}
\dot{x} \\
\ddot{x} \\
\dot{y} \\
\ddot{y}
\end{array}\right)=\left(\begin{array}{c}
\dot{x} \\
F_{x}-\left(4.7+\frac{0.75}{\sqrt{\left(\dot{x}^{2}+\dot{y}^{2}\right)}}\right) \dot{x} \\
\dot{y} \\
F_{y}-\left(4.7+\frac{0.75}{\sqrt{\left(\dot{x}^{2}+\dot{y}^{2}\right)}}\right) \dot{y}
\end{array}\right)=\left(\begin{array}{c}
z_{2} \\
F_{x}-\left(4.7+\frac{0.75}{\sqrt{\left(\mathrm{z}_{2}^{2}+z_{4}^{2}\right)}}\right) \mathrm{z}_{2} \\
z_{4} \\
F_{x}-\left(4.7+\frac{0.75}{\sqrt{\left(\mathrm{z}_{2}^{2}+z_{4}^{2}\right)}}\right) z_{4}
\end{array}\right)
$$

We note 
$U \in \mathbf{R}^{2},=\left(\begin{array}{c}F_{x} \\ F_{y}\end{array}\right)$ is the control vector composed of muscular forces signals $F_{x}$ and $F_{y}$

$f(z) \in \mathbf{R}^{4}$ is a non-linear function given by:

$f(z)=\left(\begin{array}{c}0 \\ -\left(\frac{0.75}{\sqrt{\left(\mathrm{z}_{2}^{2}+z_{4}^{2}\right)}}\right) \mathrm{z}_{2} \\ 0 \\ -\left(\frac{0.75}{\sqrt{\left(\mathrm{z}_{2}^{2}+z_{4}^{2}\right)}}\right) \mathrm{z}_{4}\end{array}\right)$

$A \in \mathbf{R}^{4 \times 4}$ is the state matrix, $A=\left(\begin{array}{cccc}0 & 1 & 0 & 0 \\ 0 & -4.7 & 0 & 0 \\ 0 & 0 & 0 & 1 \\ 0 & 0 & 0 & -4.7\end{array}\right)$

$B=\mathbf{R}^{2 \times 4}$ is the control matrix, $\left(\begin{array}{cc}0 & 0 \\ 1 & 0 \\ 0 & 0 \\ 0 & 1\end{array}\right)$

$d \in \mathbf{R}^{2}$ is the output vector, with $d=\left(\begin{array}{l}x \\ y\end{array}\right)$

$C \in \mathbf{R}^{4 \times 2}$ is the output matrix, $C=\left(\begin{array}{cccc}1 & 0 & 0 & 0 \\ 0 & 0 & 1 & 0\end{array}\right)$

The forces of two forearm muscles, activated during the writing act, characterize inputs of the handwriting model. The studied handwriting model estimated that the equivalent muscle forces, applied on the pen-tip, are loaded with two types of EMG signals, measured on the surface of the forearm during the movement of the writing. These forces succeeded in reconstructing handwriting graphic traces.

To determine the equivalent forces included in the motion equation, only the duration of the application of the forces $F_{x}$ and $F_{y}$ is considered.

Therefore, a three-level control is established, taking into account essentially the time of application of muscular forces. The position deviations relative to $x$ and $y$ axes at the instant $t_{i}$, are noted respectively, $\Delta x_{i}$ and $\Delta y_{i}$ as: $\Delta x_{i}=x_{i+1}-x_{i}$ and $\Delta y_{i}=y_{i+1}-y_{i}$

The sign of $\Delta x$ and $\Delta y$ indicates the direction of movement of the pen-tip (i.e. up, down, right, left) during the writing act:
$\operatorname{sign}\left(F_{x}\right)\left\{\begin{array}{l}\Delta x>0, F_{x}=F_{x}^{+}=1 \\ \Delta x<0, F_{x}=F_{x}^{-}=-1 \\ \Delta x=0, F_{x}=F_{x}^{0}=0\end{array}\right.$

$\operatorname{sign}\left(F_{y}\right)\left\{\begin{array}{l}\Delta y>0, F_{y}=F_{y}^{+}=1 \\ \Delta y<0, F_{y}=F_{y}^{-}=-1 \\ \Delta y=0, F_{y}=F_{y}^{0}=0\end{array}\right.$

Thus, if sign $\left(F_{x, y}\right)$ changes, it indicates a change in the direction of writing pattern (i.e. from up to a down movement or from a vertical to horizontal movement and conversely, etc.)

Fig.4(a) gives an example of muscles forces exerted by muscles during the writing of the Arabic letter "SIN" in fig.4(b). From fig.4(a) it can be seen that the muscles forces form illustrate the changing of trajectory direction during the writing act. However, the muscles forces do not provide information about the nature of the movement.
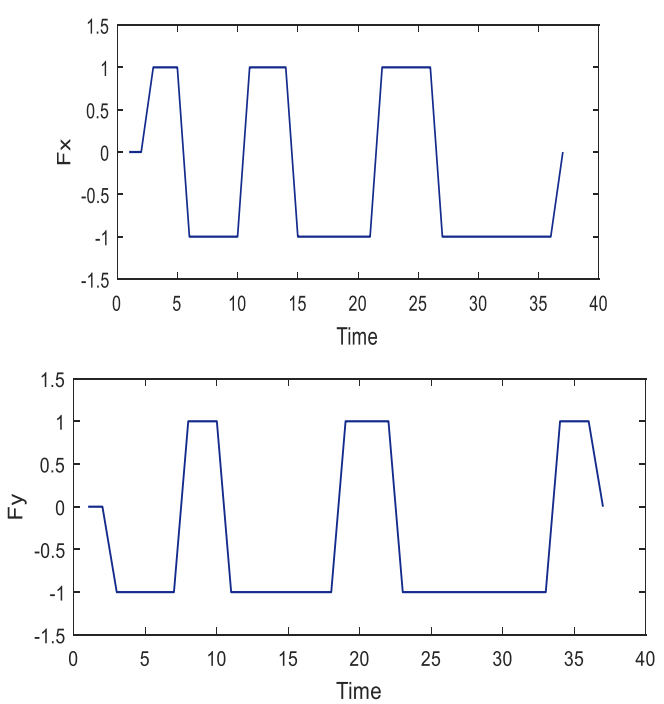

(a)

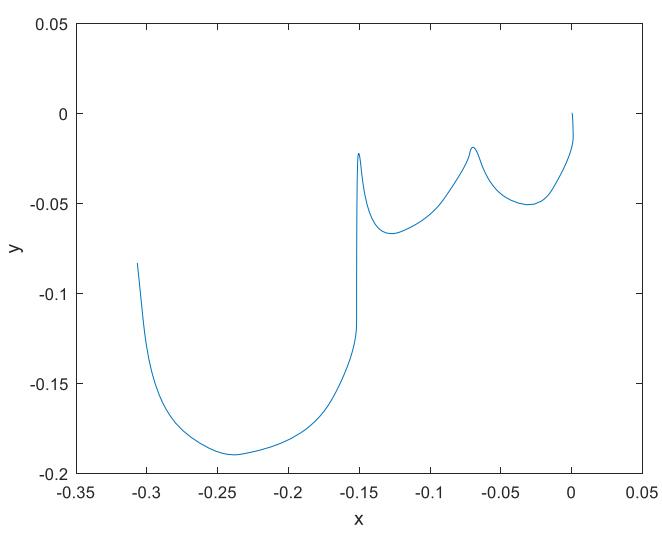

(b)

Fig. 4. Referential muscular stimuli of the Arabic Letter "SIN", (a) muscular forces, (b) Letter form 


\section{FEEDBACK CONTROLLER TO THE HANDWRITING MODEL}

In this section, we illustrate different results produced by handwriting model controller and thereafter with the proposed approach based on the feedback controller to characterize the handwriting process.

\section{A. Controller for the Handwriting System}

As described earlier for the system (4), the state space representation of handwriting system could be expressed in (8):

$\left\{\begin{array}{l}\dot{z}=A z+f(z)+B U \\ d=C z\end{array}\right.$

Firstly, for the current writing instant $t_{i}$, where $i \in \mathbb{N}$, equation (9) describe the considered model controller:

$U\left(t_{i}\right)=\operatorname{sign}(U) \times \mathrm{t}_{i}$

The figure 5 illustrates the response of the handwriting model for three Arabic letter, "SIN" in fig.5(a), "HA" in fig.5(b) and "AYN" in fig.5(c). Simulations were also realized for horizontal line in fig.5(d) and a circle in fig.5(e) and a triangle in fig.5(f). The model's outputs are represented in dashed line and the experimental recorded data by a continuous line.

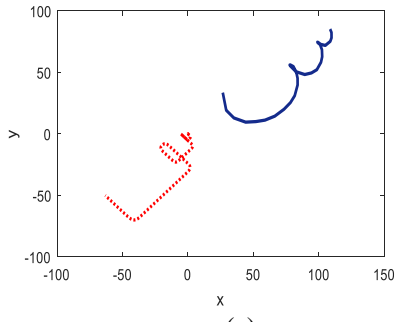

(a)

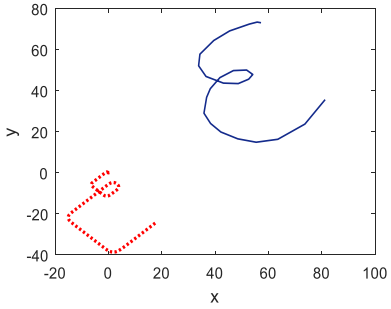

(c)

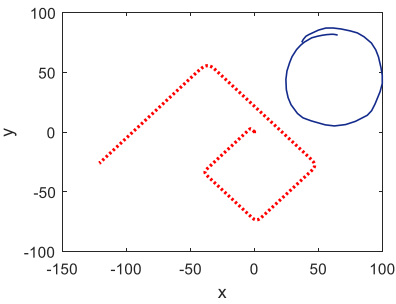

(e)

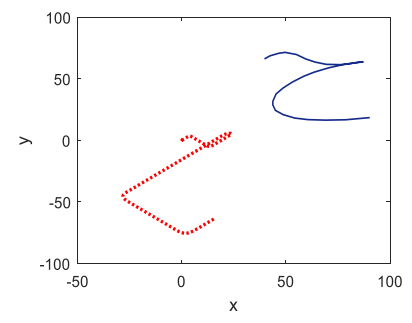

(b)

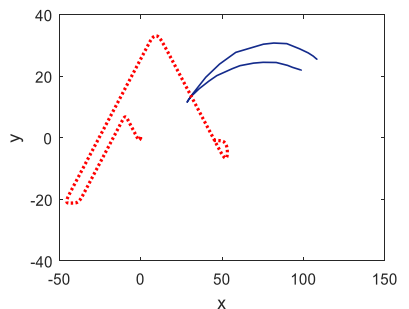

(d)

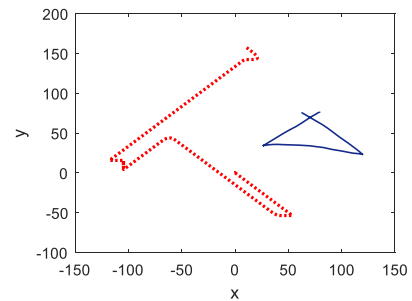

(f)
Fig. 5. Response of the handwriting model to a linear control generated from experimental database (a) Arabic Letter "SIN", (b) Arabic letter "HA", (c) Arabic letter "AYN", (d) Horizontal line, (e) Circle, (f) Triangle.
According to these simulation results, there is a considerable deviation between the experimental data and the model outputs, expressed in "(10)":

$\left\{\begin{array}{l}r_{x}\left(t_{i}\right)=x\left(t_{i}\right)-x_{e x}\left(t_{i}\right) \\ r_{y}\left(t_{i}\right)=y\left(t_{i}\right)-y_{e x}\left(t_{i}\right)\end{array}\right.$

where $d_{e x}=x_{e x}, y_{e x}$ are the coordinates of experimental recorded data and $d=x, y$ are outputs generated by the Yasuhara model.

Fig.4 illustrates errors signals according to $x$ movement presented in continuous line, and $y$ movement in dashed line. Fig.6(a) is related to Arabic letter "SIN" and fig.6(b) to Horizontal line.

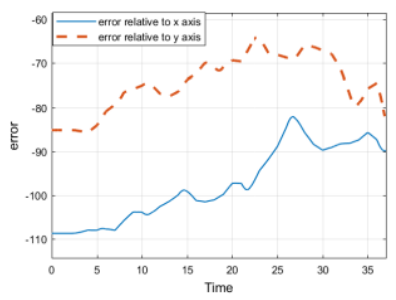

(a)

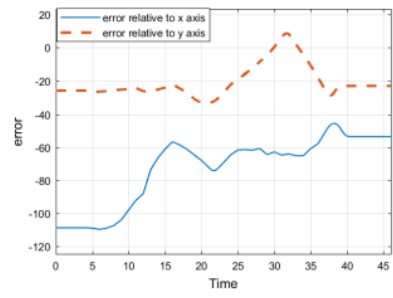

(b)
Fig. 6. Error signal according to $\mathrm{x}$ and $\mathrm{y}$ axis movements.

These results need to be improved. The purpose is to reduce the error signal $r_{x, y}$ between the model and the experimental data.

\section{B. PD feedback controller}

In this paper, the main purpose is to attempt a rapid dynamic without exceeding the order signal. The recalled information is transferred to motor control of the arm and hand to achieve the desired writing movement.

Equation "(11)" expresses the adding of a feedback controller to handwriting system

$\left\{\begin{array}{l}\dot{z}=A z+f(z)+B U^{c} \\ d=C z\end{array}\right.$

$U_{c} \in \mathbf{R}^{4 \times 2}$ Illustrated in (12) is a control expressed by current error between the experimental data and model outputs as equivalent to the memorial information recalled in the brain. With the adding of writing speed term, as the writing coordinates derivative. In this case, the control type is called PD; proportional and derivative.

$$
U^{c}=U+k_{d}\left(d_{e x}-d\right)-k_{v} v
$$

where matrices $k_{d}$ and $k_{v}$ are gain of position feedback and writing speed.

Gain matrices are chosen to satisfy the condition that the closed loop system in (13) is stable.

$$
\dot{z}=A z+f(z)+B\left(U+k_{d}\left(d_{e x}-d\right)-k_{v} v\right.
$$


For this work, we choose the gain matrix as $k_{d}=I_{2}$ and $k_{v}=I_{l}$ witch verifies the condition of model stability, and $\lim _{t \rightarrow \infty}\left(d_{e x}(t)-d(t)\right)=0$

To illustrate the results of the proposed controller, simulation of PD controller for handwriting model were performed and compared to results obtained with the 3-level controller results, given in Fig.5.

The results of simulation are illustrated in Fig.7. In these figures, model responses are presented in continuous line and the experimental data coordinates in dashed line.

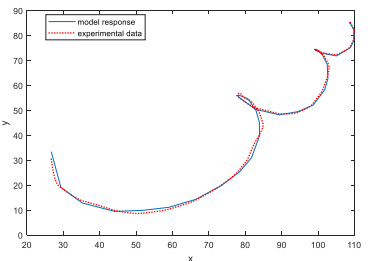

(a)Letter "SIN"

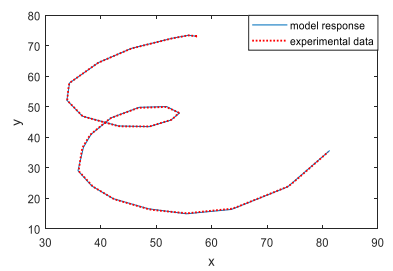

(c)Letter "AYN"

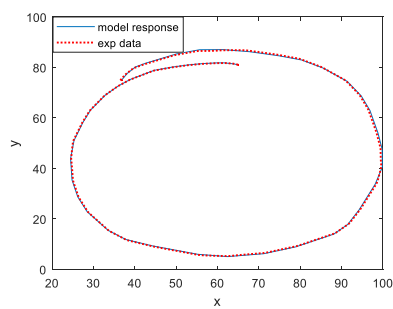

(e) Form "Circle"

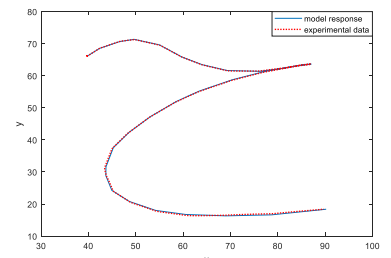

(b)Letter "HA"

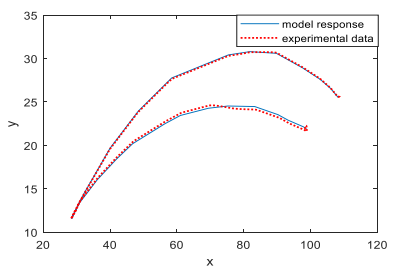

(d)Horizontal movement

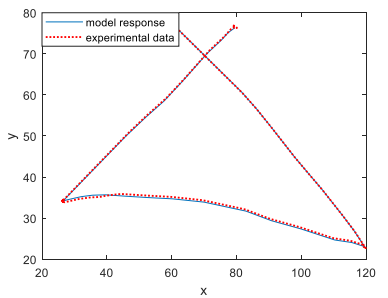

(f) Form "Triangle"
Fig. 7. Handwriting model response with feedback control.

The analysis of Fig.7 graphs show satisfactory results are obtained using a PD controller. In fact, the handwriting model response is in a good agreement with the experimental data.

For a further analysis, fig. 8 depicts simulation results of error signals of handwriting model using a PD controller, for the Arabic letter "SIN" and geometric form "Triangle".

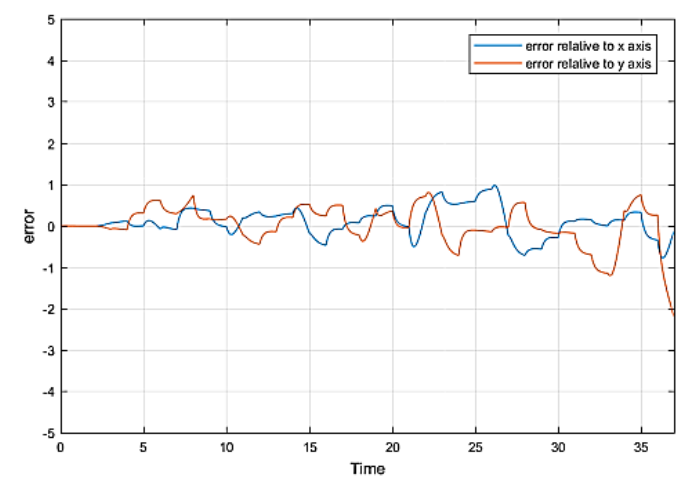

(a)

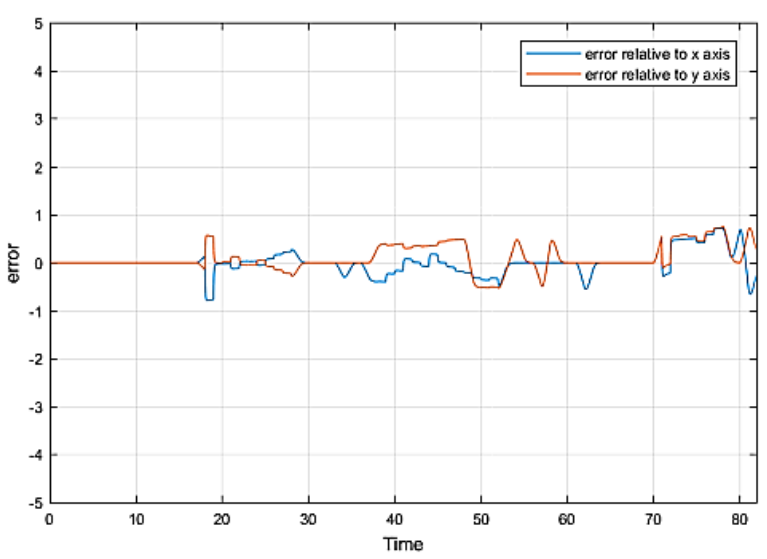

(b)

Fig. 8. Error signal form relative to $\mathrm{x}$ and $\mathrm{y}$ axis for (a) Arabic letter "SIN" and (b) Form "Triangle".

In fact, to verify this method, the error signals of Fig. 8 are compared to error signals in Fig.6. According to the analysis results, it's obvious that the error is considerably reduced and almost negligible. Thus, the model response matches the experimental data. The presented results prove that the method is an effective way to improve model stability and accuracy.

However, a question still unanswered is whether the effectiveness of this approach if we further consider four muscles for the study instead of two muscles.

\section{CONCLUSION}

The main contribution of this paper is to design a feedback controller to model the handwriting motion only from two forearm forces of muscles.

A particular attention is paid to ensure the control of writing path and make the model response following the desired trajectory. Our contribution refers to a comparing between results of handwriting model controller and the proposed solution, a PD feedback controller for the control of the handwriting model. To validate the presented approach a database of experimental recorded data is considered to perform simulation results. The relevance of this control technique is illustrated in the obtained results. The presented controller has great potential for a practical application such as a robotic hand that could mimic the human handwriting as accurately as possible.

Applying feedback control technique was always considered in field of biology and biomedical and especially for handwriting system, it is necessary to ensure the model robustness against unknown inputs and prevent model uncertainties, which will be very helpful further for studying fault detection and diagnosis issues, especially with faults attempting the actuator system.

\section{REFERENCES}

[1] I. Kuzborskij, A. Gijsberts and B. Caputo, "On the challenge of classifying 52 hand movements from surface electromyography," Annual International Conference of the IEEE Engineering in Medicine and Biology Society, San Diego, CA, pp. 4931-4937, 2012.

[2] L. Rossini and P. M. Rossini, "Combining ENG and EEG integrated analysis for better sensitivity and specificity of neuroprosthesis 
operations,"Annual International Conference of the IEEE Engineering in Medicine and Biology, Buenos Aires, pp. 134-137, 2010.

[3] T. R. D. Scott, V. A. Vare. "A novel five degree of freedom user command controller in people with spinal cord injury and non-injured for full upper extremity neuroprostheses, wearable powered orthoses and prosthesis". Med Biol Eng Comput, Vol. 51, pp. 317-30, 2013.

[4] VT. Inman, HJ Ralston, JB Saunders, B Feinstein, and EW Wright, Relation of human electromyogram to muscular tension. Electroencephalogr Clin Neurophysiol, Vol. 4, pp. 187-194, 1952.

[5] HS. Milner-Brown, and RB. Stein, The relation between the surface electromyogram and muscular force, J Physiol, Vol. 246, pp. 549-569, 1975.

[6] J. Chalfoun, M. Renault, R. Younes and F. B. Ouezdou, "Muscle forces prediction of the human hand and forearm system in highly realistic simulation, IEEE/RSJ International Conference on Intelligent Robots and Systems (IROS) Vol.2, pp. 1293-1298, Sendai, 2004.

[7] M. Rasouli, R. Ghosh, W. W. Lee, N. V. Thakor and S. Kukreja, Stable force-myographic control of a prosthetic hand using incremental learning, Annual International Conference of the IEEE Engineering in Medicine and Biology Society (EMBC), pp. 4828-4831, Milan, 2015.

[8] M. Sakr and C. Menon, Study on the force myography sensors placement for robust hand force estimation, IEEE International Conference on Systems, Man, and Cybernetics (SMC), pp. 1387-1392, Banff, AB, 2017.

[9] M. Sano, T. Kosaku and Y. Murata, Modeling of human handwriting motion by electromyographic signals on forearm muscles", CCCT'03, 2003, Orlando-Florida.

[10] M. Benrejeb and A. El Abed-Abdelkrim, "Speed analysis of handwriting process models, IEEE International Conference on Systems, Man and Cybernetics, pp. 276-281, 2002, Yasmine Hammamet.
[11] I. Chihi, A. Abdelkrim et M. Benrejeb, Internal model Control to characterize human handwriting motion, International Arab Journal of Information Technology (IAJIT), Vol. 14, no. 6, 2017.

[12] M. Yasuhara, Experimental studies of handwriting process, Rep. Univ. Electro-Comm, Vol. 25 (2), pp. 233-254, 1975.

[13] C. Chapuis, E. Bideaux, X. Brun and N. Minoiu-Enache, "Comparison of feedback linearization and flatness control for anti-slip regulation (ASR) of an hybrid vehicle: From theory to experimental results," 2013 European Control Conference (ECC), Zurich, 2013, pp. 446-451.

[14] F. Abry, X. Brun, S. Sesmat, É. Bideaux and C. Ducat, "Electropneumatic Cylinder Backstepping Position Controller Design With Real-Time Closed-Loop Stiffness and Damping Tuning," in IEEE Transactions on Control Systems Technology, vol. 24, no. 2, pp. 541552, March 2016.

[15] C. Ibtissem and L. Nouredine, "Design of optimal PD controller for inverted pendulum using differential evolution," 2013 International Conference on Control, Decision and Information Technologies (CoDIT), Hammamet, 2013, pp. 595-600.

[16] D. Salin, P.J. Arnoux, K. Kayvantash and M. Behr Implementation of reflex loops in a biomechanical finite element model, Computer Methods in Biomechanics and Biomedical Engineering, 19:14, 1578-1582, 2016.

[17] J. Östh , K. Brolin and R. Happee, Active muscle response using feedback control of a finite element human arm model, Computer Methods in Biomechanics and Biomedical Engineering, 15:4, 347-361, 2012.

[18] M. Benrejeb, A. El Abed-Abdelkrim, S. Bel Hadj Ali and M. Gasmi, A neuro-fuzzy internal model controller for handwriting process, CESA Multiconference, Lille, France, 2003.

[19] M. Benrejeb, A. Abdelkrim and M. Sano, "Sur l'étude du processus d'écriture à la main. Approches classiques et non conventionnelles," Revue e-STA, Vol. 2, 2006. 